\title{
Charge transfer and Fermi level shift in $p$-doped single-walled carbon nanotubes
}

\author{
W. Zhou, J. Vavro, N. M. Nemes,* and J. E. Fischer ${ }^{\dagger}$ \\ Department of Materials Science and Engineering, University of Pennsylvania, Philadelphia, Pennsylvania 19104, USA \\ F. Borondics and K. Kamarás \\ Research Institute for Solid State Physics and Optics, Hungarian Academy of Sciences, Budapest, Hungary \\ D. B. Tanner \\ Department of Physics, University of Florida, Gainesville, Florida 32611, USA \\ (Received 17 January 2005; revised manuscript received 21 March 2005; published 31 May 2005)
}

\begin{abstract}
The electronic properties of $p$-doped single-walled carbon nanotube (SWNT) bulk samples were studied by temperature-dependent resistivity and thermopower, optical reflectivity, and Raman spectroscopy. These all give consistent results for the Fermi level downshift $\left(\Delta E_{F}\right)$ induced by doping. We find $\Delta E_{F} \approx 0.35 \mathrm{eV}$ and $0.50 \mathrm{eV}$ for concentrated nitric and sulfuric acid doping respectively. With these values, the evolution of Raman spectra can be explained by variations in the resonance condition as $E_{F}$ moves down into the valence band. Furthermore, we find no evidence for diameter-selective doping, nor any distinction between doping responses of metallic and semiconducting tubes.
\end{abstract}

DOI: 10.1103/PhysRevB.71.205423

PACS number(s): 71.23.-k, 61.48.+c, 73.63.Fg, 81.07.De

The electronic spectra of single-wall carbon nanotubes (SWNTs) are dominated by van Hove singularities, manifestations of the 1-D structure. The location of the Fermi energy $E_{F}$ with respect to these singularities can be tuned by chemical (alkali metals, acids, halogens, ...) $)^{1}$ or electrochemical doping. ${ }^{2}$ Doping response in bulk samples is complicated by the presence of metallic and semiconducting tubes and by diameter and chirality dispersion, both of which imply a distribution of initial work functions. ${ }^{3,4}$ Further complications arise from tube-tube interactions in bundles or ropes. ${ }^{5,6}$ Here we report a systematic study of chemically $p$-doped SWNTs combining resistivity, thermopower, reflectivity, and Raman spectroscopy. Experimental results from each of the above techniques have been reported before, but quantitative analysis of the Fermi level shift has not been routinely performed. Also, the consistency of experimental results from different techniques has never been carefully addressed. In this work, we compare data obtained for relatively weak and strong protonic acids, $\mathrm{HNO}_{3}$ and $\mathrm{H}_{2} \mathrm{SO}_{4}$, respectively, in order to test consistency of results from different measurements. We discuss the results in terms of a rigid band model ${ }^{7}$ whereby doping shifts $E_{F}$ without affecting the band structure. We assume all tubes in the undoped bulk sample have the same work function, and that $E_{F}$ is initially near the middle of the gap or pseudogap of semiconducting or metallic tubes, respectively. We also assume that doping is spatially uniform, with no energy barriers between metallic and semiconducting tubes. We find that this simplest of models gives consistent results for $\Delta E_{F}$, the Fermi level shift upon doping. Using the experimentally determined $\Delta E_{F}$ values as input, the evolution of Raman spectra with doping can be simply explained by the variation of resonance conditions with $E_{F}$, with no evidence for diameter-selective doping as recently proposed. ${ }^{8-10}$

Samples were prepared from pulsed laser vaporization (PLV) ${ }^{11}$ and HiPco SWNT. ${ }^{12}$ The former have a narrow distribution of relatively large diameters, ${ }^{13} 1.36 \pm 0.09 \mathrm{~nm}$, while HiPco tubes have smaller average diameters extending over a broad range, ${ }^{14} 0.8$ to $1.4 \mathrm{~nm}$. Starting materials for the doping experiments were purified SWNT in the form of filter-deposited PLV "buckypapers,"13 solvent-cast HiPco films, ${ }^{15}$ and HiPco fibers extruded from suspensions in the superacid oleum (anhydrous $\mathrm{H}_{2} \mathrm{SO}_{4}+\mathrm{a}$ few $\% \mathrm{SO}_{3}$ ). ${ }^{16,17}$ Buckypapers and films were vacuum annealed at $1100{ }^{\circ} \mathrm{C}$ prior to doping, which was accomplished by immersion in concentrated acid overnight followed by air drying to minimize loss of dopant. Extruded fibers contain varying amounts of residual $\mathrm{H}_{2} \mathrm{SO}_{4}$ depending on the coagulation bath and are strongly $p$-type. ${ }^{16}$ High temperature annealing drives off the acid, allowing comparison with properties in the undoped state. In contrast to alkali metals, ${ }^{18}$ we found no effects of atmospheric exposure on the acid-doped samples.

We used x-ray diffraction (XRD) to investigate structural changes upon doping. The top curves in Fig. 1 show that SWNTs in PLV buckypaper exist mainly as nanocrystalline ropes $^{13}$ while HiPco SWNT film shows only weak evidence for tube-tube correlations. ${ }^{14}$ High resolution transmission electron microscopy (not shown) reveals prolific SWNT assemblies in both materials. The Bragg peaks in PLV are broadened somewhat by diameter dispersion ${ }^{19}$ and also by finite size effects. ${ }^{13}$ In contrast, most HiPco samples do not exhibit sharp features, primarily due to the larger diameter dispersion. ${ }^{14,16}$

Figure 1 shows that both acids produce drastic reductions in X-ray peak intensities for both SWNT materials. This can be understood as the combined effect of structure factor variations $^{20}$ and doping-induced structural disorder of the ropes/bundles. ${ }^{21}$ The effect of $\mathrm{H}_{2} \mathrm{SO}_{4}$ doping is notably more dramatic than $\mathrm{HNO}_{3}$ doping, implying that the former is the stronger dopant. Despite differences in initial crystallinity, peak intensities diminish progressively from undoped through nitric to sulfuric for both PLV and HiPco. This suggests that the different average diameters are not significant in the doping process. 


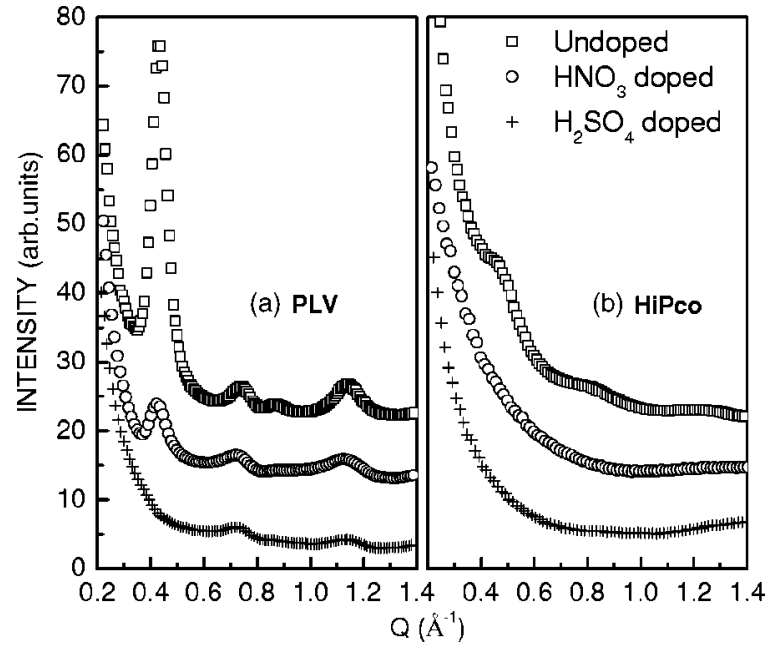

FIG. 1. The x-ray diffraction data from (a) PLV and (b) HiPco buckypapers. The PLV material is more crystalline than HiPco and has a narrower diameter distribution. The major effect of doping is the loss of Bragg intensity, more dramatic for sulfuric than nitric acid. No difference in doping response was found between PLV and HiPco, thus no evidence for diameter selectivity.

Figure 2 shows resistivity versus temperature $\rho(T)$ for a PLV buckypaper. Before doping, $\rho(300 \mathrm{~K}) \sim 10 \mathrm{~m} \Omega \mathrm{cm}$ and the resistance ratio $\rho(1.6 \mathrm{~K}) / \rho(300 \mathrm{~K}) \sim 6$ is nonmetallic. Doping with either acid leads to dramatic reduction in $\rho$ at all $T$, and weaker temperature dependence; sulfuric acid doping leads to metallic behavior, $d \rho / d T>0$, above $100 \mathrm{~K}$. HiPco fibers behave similarly, except that the pristine fibers are metallic from residual oleum doping while the nonmetallic state is achieved by vacuum annealing. ${ }^{15}$ Results from PLV and HiPco are thus consistent with respect to doped versus undoped states.

The nonmetallic behavior in the undoped state, with diverging $\rho(T)$ as $T>0$, is due to localization of charge carriers. ${ }^{22} E_{F}$ is near the neutrality point; at low $T$ semicon-

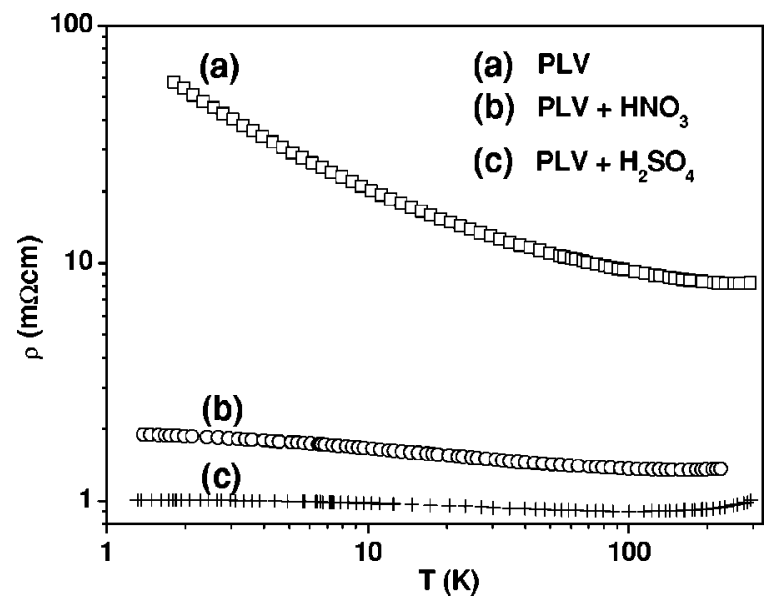

FIG. 2. (Color online) The doping effect on resistivity and its temperature dependence. Doping reduces $\rho(300 \mathrm{~K})$, approximately twice as much for sulfuric than nitric acid. The temperature dependence becomes weaker after doping, more dramatically so for sulfuric than nitric acid.

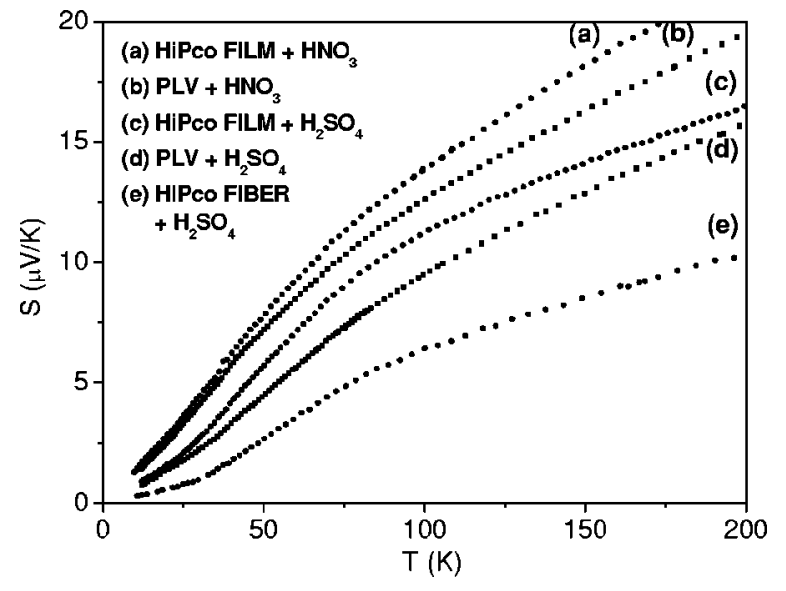

FIG. 3. (Color online) The doping effect on thermopower. For undoped and air-exposed SWNT S(T) increases monotonically to $60-80 \mu \mathrm{V} / \mathrm{K}$ at $300 \mathrm{~K}$ (not shown). Doping reduces the amplitude at all $T$ and introduces an inflection point at characteristic temperatures in the range $20-50 \mathrm{~K}$ for different samples. Both effects are most apparent in the $\mathrm{H}_{2} \mathrm{SO}_{4}$-doped HiPco fiber.

ducting tubes of all band gaps are insulating, and coupling between metallic tubes is poor. Upon $p$-doping, $E_{F}$ approaches the valence band maxima of semiconducting tubes, leading eventually to carrier degeneracy and metallic behavior down to the lowest temperatures. The resistivity is considerably reduced by two mechanisms; previously semiconducting tubes now contribute to conduction, and coupling between tubes is enhanced. The doped bulk sample behaves like a disordered metal with finite $\rho$ at $T=0$. From Fig. 2 we conclude that $\mathrm{H}_{2} \mathrm{SO}_{4}$ is a stronger oxidative dopant than $\mathrm{HNO}_{3}$, and therefore $E_{F}$ is shifted further down into the valence band compared to $\mathrm{HNO}_{3}$ doping. The same applies to HiPco-derived films and fibers (not shown).

Another property strongly dependent on doping is the thermopower or Seebeck coefficient $S$. While $S$ of airexposed but otherwise undoped SWNTs is not very well understood, low-temperature $S$ of chemically doped samples can be accounted for very well by a dominant phonon drag contribution $S_{g} .{ }^{23}$ Due to the 1-D electron spectra and linear $E(k)$ dispersion of metallic SWNTs, only phonons with wave vector $Q \geqslant 2 E_{F} / \hbar v_{F}$ contribute to electron-phonon interactions at low $T\left(v_{F}\right.$ is the electron group velocity). This leads to $S_{g}(T) \propto C_{p h}\left(\hbar \omega_{Q} / k_{B} T\right)$, where $C_{p h}\left(\hbar \omega_{Q}\right)$ is the heat capacity contribution from a phonon with wave vector $Q$. All tubes are metallic after doping, so the total low- $T$ thermopower is the sum of phonon drag and carrier diffusion terms, $S=S_{g}+b T$ for a single tube type. The characteristic features of $S(T)$ are a phonon drag threshold at $k_{B} T_{0} \approx 0.1 \hbar \omega_{Q}$ and a maximum slope at $2.3 T_{0} . E_{F}$ is obtained by fitting $S(T)$ data to $a C_{p h}\left(\hbar \omega_{Q} / k_{B} T\right)+b T$, which gives $Q$, then evaluating $\left|E_{F}\right|=\left(v_{F} / 2 v_{p h}\right) \hbar \omega_{Q}=29.1 \hbar \omega_{Q}$. We use the twiston sound velocity ${ }^{24}$ for $v_{p h}$. Data for several doped samples are shown in Fig. 3. The inflection points (located from numerical derivatives) all lie in the range $20-50 \mathrm{~K}$, most prominently for the HiPco fiber which has the highest value of $T_{0}$. There is no inflection point for undoped tubes. These results are qualitatively consistent with $\rho$ data; sulfuric 
acid gives the greater $E_{F}$ downshift and the lower $\rho$ at all $T$ compared to nitric acid.

The increased carrier concentration and $E_{F}$ downshift upon doping imply detectable changes in the free-carrier plasma edge and van Hove interband transitions, respectively. The latter have been thoroughly studied by electron energy loss (EELS $)^{25}$ and optical absorption. ${ }^{26}$ Reflectance spectroscopy is a convenient method to study both phenomena. A wide range of photon energies can be explored without evacuating the instrument, a prerequisite for materials doped with volatile acceptors.

Absorption spectroscopy shows that $n$ - and $p$-doping both lead to reduced intensities of the first and second van Hove transitions as the relevant initial or final state becomes occupied or emptied. ${ }^{26}$ The first reports of nanotube reflectance spectroscopy showed a very broad plasma edge around $0.1 \mathrm{eV}$, with no shift after potassium doping. ${ }^{27,28}$ Interband transitions and their polarization dependence were studied in undoped oriented samples by Hwang et al. ${ }^{29}$ We previously reported a systematic study of plasma edge shifts and interband transition quenching in alkali-doped foils sealed in evacuated cuvettes; ${ }^{30}$ the data revealed much sharper features and systematic plasma edge shifts with alkali concentration.

In this work we use near-infrared and visible reflectance spectroscopy to study the doping dependence of free-carrier and interband processes on bare samples of PLV and HiPco foils doped with nitric and sulfuric acids. Surface scattering corrections were made by measuring the reflectance of a matching buckypaper coated with aluminum. ${ }^{30}$ Spectra were fitted to a model dielectric function consisting of a free carrier Drude term, Lorentz oscillators representing interband van Hove transitions, and a background function accounting for screening by higher energy transitions. For the latter we used the dielectric function derived from EELS on similar undoped SWNTs, ${ }^{31}$ along with a small adjustable constant to account for doping-induced variations in the $\pi$ and $\pi+\sigma$ plasmons.

The data and fits are shown in Figs. 4(a) and 4(b) for PLV and HiPco, respectively. Undoped samples could be exposed to vacuum, so their profiles extend down to $0.01 \mathrm{eV}$. The blueshift of the plasma minimum from $0.3 \mathrm{eV}$ to as high as $0.9 \mathrm{eV}$, as well of the quenching of the first interband transition at $\sim 0.8 \mathrm{eV}$, are dramatic. Differences between the two acids, and the two nanotube sources, are easily discerned; all the features in reflectivity spectra are sharper in PLV compared to HiPco, and both doping effects are greater with sulfuric than with nitric acid. These qualitative trends are entirely consistent with XRD, conductivity, and thermopower. Details emerge from the curve fits summarized in Table I. In the spirit of the rigid band model, positions and widths of van Hove transitions were fixed at the fitted undoped values, and the $E_{F}$ shifts with doping were inferred from the fitted oscillator strengths, which decrease as successive valence band singularities become unoccupied. The fit parameters describing van Hove transitions and the freecarrier contribution are summarized in Table I. For both the PLV and HiPco series, the same trends are observed. With doping, the first van Hove transition diminishes for $\mathrm{HNO}_{3}$ doping and disappears for $\mathrm{H}_{2} \mathrm{SO}_{4}$ doping, and the intensities

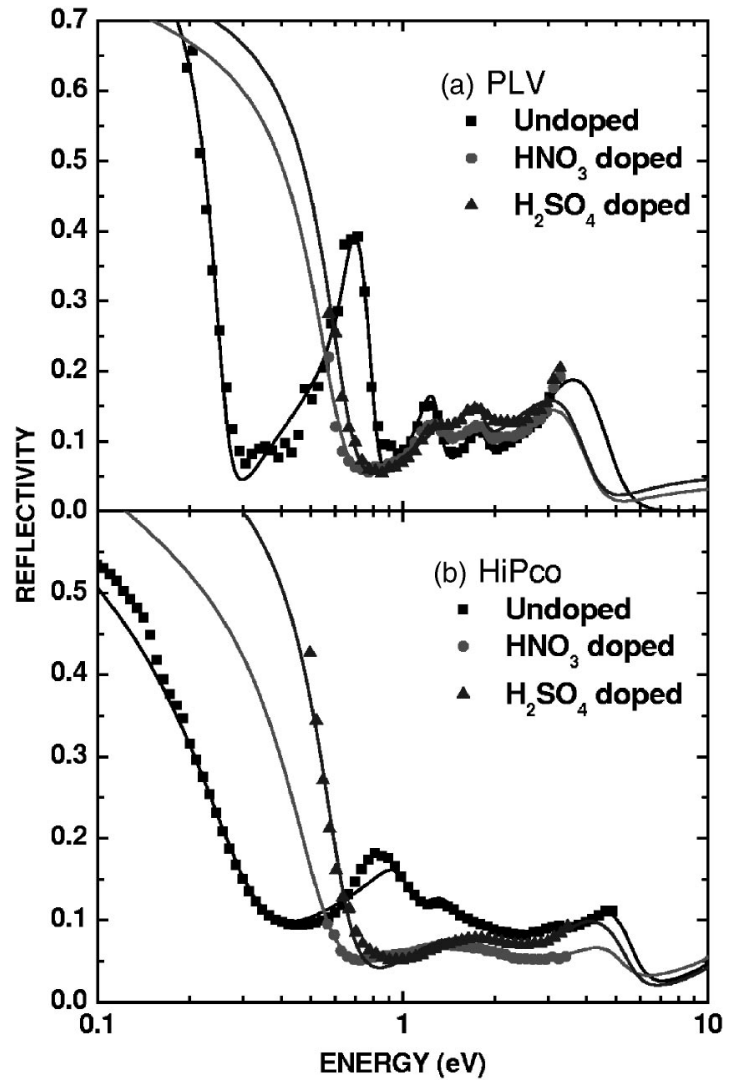

FIG. 4. (Color online) The reflectivity and Drude-Lorentz fits for undoped, $\mathrm{HNO}_{3}$-doped, and $\mathrm{H}_{2} \mathrm{SO}_{4}$-doped PLV (a) and HiPco (b) buckypapers. Two systematic effects are observed: a blueshift of the reflectivity minimum (increasing Drude plasma frequency) and decreased intensity of the peaks at higher energy (quenching interband transitions by shifting $E_{F}$ ). See Table I for model fit parameters (solid curves).

of the second van Hove transition get progressively smaller as well.

Using the values of the van Hove transition energies, their widths, and the decrease in the oscillator strengths for each transition with doping, we can estimate lower and upper limits of the $E_{F}$ shift. For the PLV sample, the transition completely disappears with $\mathrm{HNO}_{3}$ doping and the second transition intensity also decreases, so we estimate $E_{F}$ to lie between the bottom of the first and the top of the second originally filled van Hove bands.

Shifts estimated from interband transition quenching are summarized in the bottom row of Table II for the four doped samples. These estimates are slightly larger than the more precise values derived from thermopower and the estimates from Raman spectroscopy to be presented below. Firstprinciples calculations ${ }^{32}$ show that the estimated change in the peak positions of the van Hove singularities of the conduction band in potassium-doped SWNT is on the order of $0.1 \mathrm{eV}$. Recent photoemission experiments on potassiumdoped SWNTs ${ }^{33}$ find that the valence band singularities of metallic SWNTs move closer to those of semiconductors. Both effects decrease the transition energy between valence and conduction bands that are observed in the optical spectra. $\Delta E_{F}$ based on the quenching of the van Hove transitions 
TABLE I. Analysis of reflectivity data: fitted values from a model containing a Drude term (free carriers), Lorentz oscillators (interband transitions), and a high frequency extrapolation derived from EELS data (Ref. $30)$.

\begin{tabular}{lccccc}
\hline \hline & Undoped & $\begin{array}{c}\text { PLV } \\
\text { nitric }\end{array}$ & $\begin{array}{c}\text { PLV } \\
\text { sulfuric }\end{array}$ & $\begin{array}{c}\text { HiPco } \\
\text { nitric }\end{array}$ & $\begin{array}{c}\text { HiPco } \\
\text { sulfuric }\end{array}$ \\
\hline $\begin{array}{l}\text { Free carrier plasma } \\
\text { frequency (eV) }\end{array}$ & 0.62 & 1.27 & 1.43 & 1.02 & 1.22 \\
\hline $\begin{array}{l}\text { Oscillator strength of } E_{11}, E_{22} \\
\text { (arbitrary units) }\end{array}$ & 0.86 & 0.0 & 0.0 & 0.26 & 0.0 \\
& 0.83 & 0.60 & 0.37 & 0.38 & 0.26 \\
\hline \hline
\end{tabular}

as observed in IR spectroscopy is only an estimate due to the discrete energy spacing of these transitions. Nevertheless, taking into account the offset introduced by doping-induced changes of the band-structure which are ignored by the rigidband model decreases our spectroscopy-based estimate of $\Delta E_{F}$, bringing the values closer to estimates based on TEP and Raman. The blueshift of the plasma edge is clearly due to doping-induced hole carriers. In a simple Drude model the unscreened plasma frequency $\omega_{\mathrm{P}}$ is related to the carrier concentration via $\omega_{\mathrm{P}}^{2}=4 \pi p e^{2} / \mathrm{m}^{*}$, where $p$ is the hole concentration and $m^{*}$ is the effective mass. From Table I the plasma frequency for sulfuric acid-doped PLV is $1.43 \mathrm{eV}$, which gives $\sim 1$ delocalized hole per 6 carbon atoms if $m^{*}=m_{0}$. Estimates of $m^{*}$ for metallic tubes are as low as $0.1 m_{0}$, in which case the charge per carbon is more in line with the saturation value $\sim 1 / 20$ for graphite bisulfate. ${ }^{34}$

Raman scattering is widely used to study doping of nanotubes. ${ }^{35}$ The distinctive aspect of this technique is that each tube type in bulk samples has a unique Raman resonance behavior due to diameter- and symmetry-dependent interband transitions. Doped SWNTs lose resonance enhancement via quenching of interband absorption by the shift of $E_{F}$, which again is different for each individual tube type. The interpretation of Raman spectra from bulk-doped samples is thus not straightforward.

The Raman intensity from an $(n, m)$ tube is proportional to a resonance factor that depends on $(n, m)$ and the laser energy $E_{\text {laser. }}$ This factor is huge (hundreds to thousands) when an allowed interband transition energy $E_{k k}(n, m)$ is close to $E_{\text {laser }}$ (the integer $k$ labels the van Hove singularities, whose energies depend on $n$ and $m$ ). If $E_{k k}$ is not close to $E_{\text {laser }}$, or if the $k k$ transition is quenched by departures from fully occupied/unoccupied initial/final states, this factor reduces to unity. Single tube measurements clearly show that the loss of resonance, or the quenching of interband absorption, takes place gradually and continuously as a function of $E_{F}$ shift. ${ }^{4}$ Furthermore, metallic and semiconducting tubes do not lose resonance in the same manner. For doped metallic tubes, $\frac{1}{2} E_{11}^{M}$ is usually much larger than $\Delta E_{F}$ (i.e., $E_{F}$ is still between $c_{1}$ and $v_{1}$ ), thus the loss of resonance is continuous and gradual versus $E_{F}$ shift (filling or depleting electron states). For doped semiconducting tubes, charge transfer begins only when $E_{F}$ shifts outside the range bounded by $c_{1}$ and $v_{1}$. Hence the loss of resonance is minor as $E_{F}$ first moves away from neutrality; once $E_{F}$ crosses $c_{1}$ (donors) or $v_{1}$ (acceptors), resonance is lost severely.

With all this in mind, we can further compare resonance loss of tubes with different diameters. Large tubes have small and closely spaced $E_{k k}$ 's compared to small ones. Regardless of which $E_{k k}$ is resonant in the undoped state, the transitions are quenched faster for a given $\Delta E_{F}$ or doped state. This is true for both metallic and semiconducting tubes. Furthermore, undoped tubes with different $(n, m)$ will have different initial resonance factors, such that upon doping the $(n, m)$ 's that were in strongest resonance will lose it faster than those initially in weaker resonance. The ideal way to probe all these features and quantify $\Delta E_{F}$ would be to create 3D maps (intensity versus shift versus $\left.E_{\text {laser }}\right)^{36}$ as a function of dopant type and concentration. Our goal here is more modest: using $\Delta E_{F}$ from, e.g., thermopower as input, to seek consistency with the shifts inferred from the doping dependence of Raman resonance.

In Fig. 5 we show Raman spectra from PLV buckypapers using $2.41 \mathrm{eV}$ (a) and $1.96 \mathrm{eV}$ (b) laser excitations. Due to the narrow diameter distribution, only $E_{33}^{S}$, the third transition in semiconducting tubes, is resonant under $2.41 \mathrm{eV}$ excitation; similarly only $E_{11}^{M}$ is resonant at $E_{\text {laser }}=1.96 \mathrm{eV}$. All the Raman features, measured with both laser energies, lose

TABLE II. Summary of doping-induced $E_{F}$ downshifts measured by different techniques. $E_{F}$ values from Raman were estimated by matching our spectra with similar data from a HiPco sample in Ref. 9.

\begin{tabular}{|c|c|c|c|c|c|c|}
\hline & $\begin{array}{c}\mathrm{PLV} \\
+\mathrm{HNO}_{3}\end{array}$ & $\begin{array}{c}\mathrm{PLV} \\
+\mathrm{H}_{2} \mathrm{SO}_{4}\end{array}$ & $\begin{array}{l}\text { HiPco } \\
\text { fiber- } \\
\text { "high" }\end{array}$ & $\begin{array}{l}\text { HiPco } \\
\text { fiber- } \\
\text { "low" }\end{array}$ & $\begin{array}{c}\mathrm{HiPco} \\
+\mathrm{HNO}_{3}\end{array}$ & $\begin{array}{c}\mathrm{HiPco} \\
+\mathrm{H}_{2} \mathrm{SO}_{4}\end{array}$ \\
\hline$E_{F}(\mathrm{eV})$ (Seebeck) & 0.33 & 0.46 & 0.53 & 0.2 & 0.35 & 0.46 \\
\hline$E_{F}(\mathrm{eV})($ Raman $)$ & & & $0.5-0.7$ & $0.2-0.3$ & & \\
\hline$E_{F}(\mathrm{eV})$ (Reflectivity) & $0.32-0.51$ & $0.51-0.62$ & & & $0.43-0.5$ & $0.6-0.75$ \\
\hline
\end{tabular}




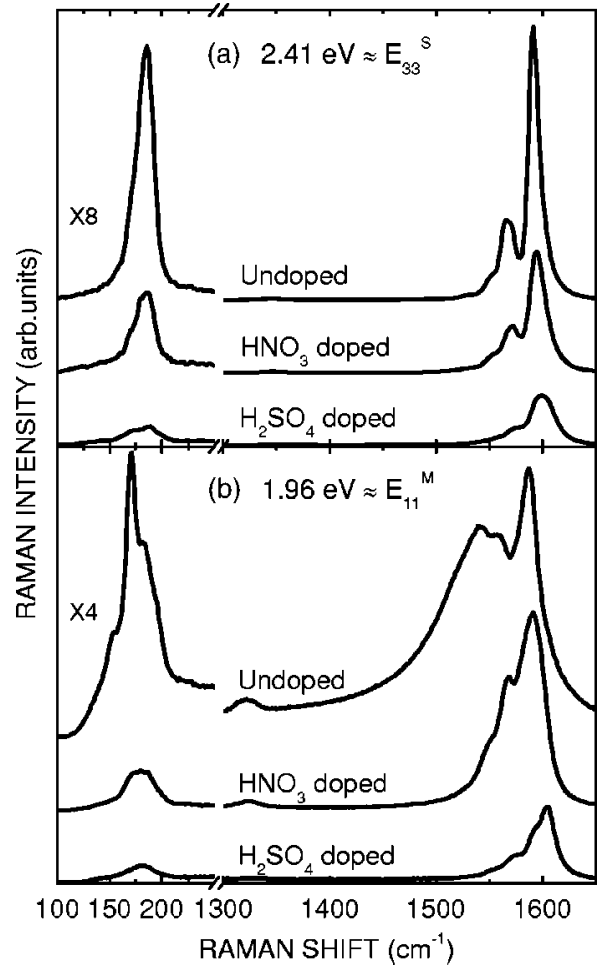

FIG. 5. The Raman spectra of undoped, $\mathrm{HNO}_{3}$-doped, and $\mathrm{H}_{2} \mathrm{SO}_{4}$-doped PLV buckypapers using (a) $2.41 \mathrm{eV}$ and (b) $1.96 \mathrm{eV}$ laser excitation. The RBM bands are magnified for clarity. Raman intensities decrease upon doping, more dramatically for sulfuric acid.

intensity upon doping. As summarized in Table $\mathrm{I}$, the estimated $\Delta E_{F}$ values for $\mathrm{HNO}_{3^{-}}$and $\mathrm{H}_{2} \mathrm{SO}_{4}$-doped PLV are 0.33 and $0.53 \mathrm{eV}$, respectively. These are less than $\frac{1}{2} E_{\text {laser }} \cong \frac{1}{2} E_{11}^{M} \cong \frac{1}{2} E_{33}^{S}$, thus the resonance is not totally quenched. The blueshift of the $G$ band upon $p$-doping is the inverse of the redshift observed in $n$-doped SWNT, ${ }^{37}$ and results from the contraction and stiffening of $\mathrm{C}-\mathrm{C}$ bonds. It is interesting to note that doping diminishes the sensitivity of Raman spectra to the choice of $E_{\text {laser }}$ since the resonance enhancement is strongly damped.

In Fig. 6 we show spectra from HiPco fibers using $2.41 \mathrm{eV}$ (a), $1.96 \mathrm{eV}$ (b), and $1.59 \mathrm{eV}$ (c) excitations. Here "light" and "heavy" doping correspond to different coagulation baths as the oleum-suspended tubes emerge as fibers from an orifice. ${ }^{16}$ In both fibers the dopant is $\mathrm{H}_{2} \mathrm{SO}_{4}$, while the different baths control their concentrations. $\Delta E_{F}$ for these fibers was determined from thermopower as described above: -0.2 and $-0.53 \mathrm{eV}$, respectively. Consistent with the previous discussion, under $2.41 \mathrm{eV}$ excitation, metallic tubes with $0.9<d<1.1 \mathrm{~nm}$ and semiconducting tubes with $1.2<d<1.6 \mathrm{~nm}$ are in resonance due to allowed interband transitions $E_{11}^{M}$ and $E_{33}^{S}$. Going from neutral to lightly doped and then heavily doped, the $185 \mathrm{~cm}^{-1}$ component loses intensity very fast and the $262 \mathrm{~cm}^{-1}$ component loses intensity more gradually. The initial $E_{F}$ is closest to $v_{1}$ of large semiconducting tubes, which thus suffer a major loss of resonance as $E_{F}$ begins to downshift (light doping). With $1.96 \mathrm{eV}$ excitation, semiconducting tubes with $0.8<d<1.0 \mathrm{~nm}$ and

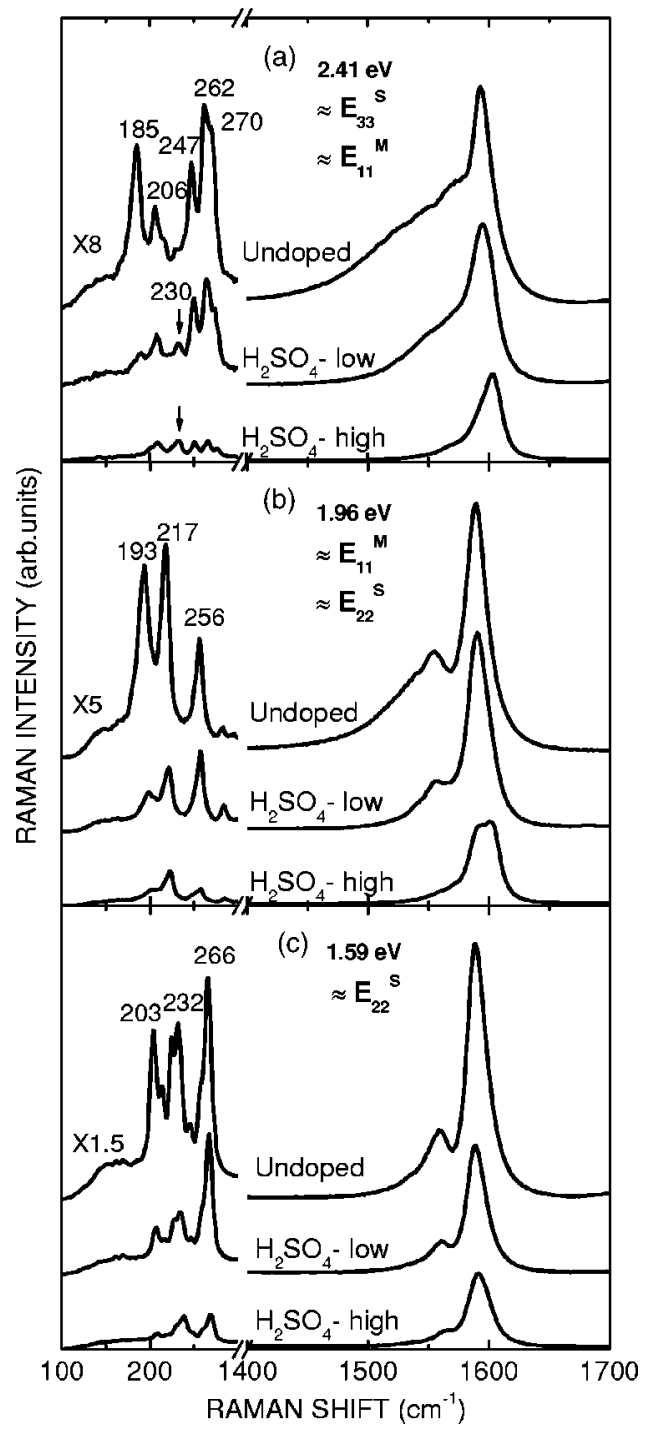

FIG. 6. The Raman spectra of HiPco fibers using (a) $2.41 \mathrm{eV}$, (b) $1.96 \mathrm{eV}$, and (c) $1.59 \mathrm{eV}$ laser excitation. The RBM bands are magnified for clarity. "Low" and "high" concentrations of $\mathrm{H}_{2} \mathrm{SO}_{4}$ were obtained using different coagulation baths in the spinning process. The loss of resonance upon doping is similar to PLV (Fig. 5). See Table II for estimated $E_{F}$ shifts associated with loss of Raman resonance.

metallic tubes with $1.2<d<1.6 \mathrm{~nm}$ are in resonance due to allowed interband transitions $E_{22}^{S} \approx E_{11}^{M} \approx 1.96 \mathrm{eV}$. After light doping $E_{F}$ still lies well above the $v_{1}$ 's of this group of semiconducting nanotubes, which thus lose resonance to a lesser degree than large metallic tubes. Subsequent $E_{F}$ downshift below $E_{11}^{S}$ leads to severe intensity loss from smaller semiconducting tubes. In contrast, for $1.59 \mathrm{eV}$ excitation only semiconducting tubes with $0.8<d<1.2 \mathrm{~nm}$ are resonant, so the only systematic trend is the loss of resonant response from large tubes with minimal doping.

In principle, tubes of different $(n, m)$ are resonant for the three laser energies. Our data show that for light doping the largest metallic and semiconducting tubes lose resonance more severely than smaller ones. For heavy doping the small tubes also lose intensity severely. Tubes of intermediate di- 
ameter are less affected at all doping conditions. We believe that these observations are all attributable to diameterdependent Raman resonance conditions. When $\Delta E_{F}$ becomes large enough, comparable to half the largest laser energy, the resonance factor approaches unity for all $(n, m)$ and the spectrum more closely reflects the diameter distribution. Indeed, the overall spectra and relative intensities for heavily doped PLV and HiPco are nearly independent of laser energy, cf. Figs. 5 and 6, despite their huge differences in the undoped state. In particular, the RBM peaks directly reflect the diameter distribution after doping because the intensity is now more sensitive to the $(n, m)$ distribution than $\Delta E_{F}$ and the resonance condition.

In a previous study, ${ }^{8} 9$ similar observations were interpreted as diameter-selective doping. It was proposed that $1-1.2 \mathrm{~mm}$ diameter tubes are less effectively doped than larger or smaller ones because access by dopant atoms or molecules to interstitial channels in the rope lattice ${ }^{11}$ depends on tube diameter. This implies that $\Delta E_{F}$ for intermediate diameters is smaller than for the others. This structure-based argument is inconsistent with the doping dependence of the RBM's. For example, the $230 \mathrm{~cm}^{-1}$ component does not lose much intensity under $2.41 \mathrm{eV}$ while the $232 \mathrm{~cm}^{-1}$ component loses intensity dramatically under $1.56 \mathrm{eV}$; both components represent tubes with similar intermediate diameters.

It was also recently proposed that some dopants may se- lectively dope either metallic SWNTs or semiconducting SWNTs in certain cases. ${ }^{38}$ These provided potential routes to sort nanotubes by diameter or electronic structure. In our acid-doped samples, we did not observe any hint of such selectivity although this does not rule out the possibility with other dopants.

In summary, we studied the charge transfer in chemically $p$-doped SWNTs combining resistivity, thermoelectric power, reflectivity, and Raman spectroscopy. We obtained consistent results from all these techniques, and quantified the Fermi level shift $\left(\Delta E_{F}\right)$ in doped samples. With the $\Delta E_{F}$ values obtained from other techniques, we are able to analyze the Raman spectra more precisely. The evolution of Raman spectra upon chemical doping is well accounted for by changes of Raman resonance condition. We found no evidence that the doping is selective with respect to tube diameter or metallic/semiconducting character.

This work was supported by ONR Grant No. N00014-031-0890 (W.Z.); USDOE Grant No. DE-FG02-98ER45701 (J.V.); NSF Grant Nos. DMR97-30298 (N.N.), OTKA T034198 (B.F., K.K.), NSF-MTA-OTKA N31622, and NSFINT 9902050 (B.F., K.K., D.B.T.); and by a grant jointly funded by NSF DMR-0305043 and DOE DE-AI0203ER46070 (D.B.T.). We thank Yury Gogotsi for the use of Drexel's Raman facility.
*Current address: CSIC, Inst Ciencia Mat, Madrid, E-28049 Spain.

†Email address: fischer@ seas.upenn.edu

${ }^{1}$ J. E. Fischer, Acc. Chem. Res. 35, 1079 (2002).

${ }^{2}$ L. Kavan, P. Rapta, L. Dunsch, M. J. Bronikowski, P. Willis, and R. E. Smalley, J. Phys. Chem. B 105, 10764 (2001).

${ }^{3}$ P. G. Collins and A. Zettl, Phys. Rev. B 55, 9391 (1997).

${ }^{4}$ K. I. Okazaki, Y. Nakato, and K. Murakoshi, Phys. Rev. B 68, 035434 (2003)

${ }^{5}$ A. A. Maarouf, C. L. Kane, and E. J. Mele, Phys. Rev. B 61, 11156 (2000).

6 "Bundles" consist of van der Waals-bound tube assemblies with no long-range intertube order. We reserve the term "ropes" for ordered assemblies exhibiting clear signatures of a 2-D triangular lattice.

${ }^{7}$ S. Kazaoui, N. Minami, R. Jacquemin, H. Kataura, and Y. Achiba, Phys. Rev. B 60, 13339 (1999).

${ }^{8}$ A. Kukovecz, T. Pichler, R. Pfeiffer, and H. Kuzmany, Chem. Commun. (Cambridge) 16, 1730 (2002).

${ }^{9}$ L. Kavan and L. Dunsch, Nano Lett. 3, 969 (2003).

${ }^{10}$ T. Pichler, A. Kukovecz, H. Kuzmany, and H. Kataura, Synth. Met. 135, 717 (2003).

${ }^{11}$ A. Thess, R. Lee, P. Nikolaev, H. Dai, P. Petit, J. Robert, C. Xu, H. Lee, S. G. Kim, D. T. Colbert, G. Scuseria, D. Tomanek, J. E. Fischer, and R. E. Smalley, Science 273, 483 (1996).

${ }^{12}$ M. J. Bronikowski, P. A. Willis, D. T. Colbert, K. A. Smith, and R. E. Smalley, J. Vac. Sci. Technol. B 19, 1800 (2001).

${ }^{13}$ A. G. Rinzler, J. Liu, P. Nikolaev, C. B. Huffman, F. J. Rodriguez-Macias, P. J. Boul, A. H. Lu, D. Heymann, D. T. Colbert, R. S. Lee, J. E. Fischer, A. M. Rao, P. C. Eklund, and R.
E. Smalley, Appl. Phys. A: Mater. Sci. Process. 67, 29 (1998).

${ }^{14}$ W. Zhou, Y. H. Ooi, R. Russo, P. Papanek, D. E. Luzzi, J. E. Fischer, M. J. Bronikowski, P. A. Willis, and R. E. Smalley, Chem. Phys. Lett. 350, 6 (2001).

${ }^{15}$ T. V. Sreekumar, T. Liu, S. Kumar, L. M. Ericson, R. H. Hauge, and R. E. Smalley, Chem. Mater. 15, 175 (2003).

${ }^{16}$ W. Zhou, J. Vavro, C. Guthy, K. I. Winey, J. E. Fischer, L. M. Ericson, S. Ramesh, R. Saini, V. A. Davis, C. Kittrell, M. Pasquali, R. H. Hauge, and R. E. Smalley, J. Appl. Phys. 95, 649 (2004).

${ }^{17}$ L. M. Ericson, H. Fan, H. Q. Peng, V. A. Davis, W. Zhou, J. Sulpizio, Y. H. Wang, R. Booker, J. Vavro, C. Guthy, A. N. G. Parra-Vasquez, M. J. Kim, S. Ramesh, R. K. Saini, C. Kittrell, G. Lavin, H. Schmidt, W. W. Adams, W. E. Billups, M. Pasquali, W. F. Hwang, R. H. Hauge, J. E. Fischer, and R. E. Smalley, Science 305, 1447 (2004).

${ }^{18}$ R. S. Lee, H. J. Kim, J. E. Fischer, J. Lefebvre, M. Radosavljevic, J. Hone, and A. T. Johnson, Phys. Rev. B 61, 4526 (2000).

${ }^{19}$ S. Rols, R. Almairac, L. Henrard, E. Anglaret, and J. L. Sauvajol, Eur. Phys. J. B 10, 263 (1999).

${ }^{20}$ J. E. Fischer, A. Claye, and R. S. Lee, Mol. Cryst. Liq. Cryst. Sci. Technol., Sect. A 340, 737 (2000).

${ }^{21}$ A. S. Claye, J. E. Fischer, C. B. Huffman, A. G. Rinzler, and R. E. Smalley, J. Electrochem. Soc. 147, 2845 (2000).

${ }^{22}$ J. Vavro, J. M. Kikkawa, and J. E. Fischer Phys. Rev. B 71, 155410 (2005).

${ }^{23}$ J. Vavro, M. C. Llaguno, J. E. Fischer, S. Ramesh, R. K. Saini, L. M. Ericson, V. A. Davis, R. H. Hauge, M. Pasquali, and R. E. Smalley, Phys. Rev. Lett. 90, 065503 (2003). 
${ }^{24}$ C. L. Kane and E. J. Mele, Phys. Rev. Lett. 90, 207401 (2003).

${ }^{25}$ T. Pichler, M. Knupfer, M. S. Golden, J. Fink, A. Rinzler, and R. E. Smalley, Phys. Rev. Lett. 80, 4729 (1998).

${ }^{26}$ H. Kataura, Y. Kumazawa, Y. Maniwa, I. Umezu, S. Suzuki, Y. Ohtsuka, and Y. Achiba, Synth. Met. 103, 2555 (1999).

${ }^{27}$ A. Ugawa, A. G. Rinzler, and D. B. Tanner, Phys. Rev. B 60, R11 305 (1999).

${ }^{28}$ B. Ruzicka, L. Degiorgi, R. Gaal, L. Thien-Nga, R. Bacsa, J. P. Salvetat, and L. Forro, Phys. Rev. B 61, R2468 (2000).

${ }^{29}$ J. Hwang, H. H. Gommans, A. Ugawa, H. Tashiro, R. Haggenmueller, K. I. Winey, J. E. Fischer, D. B. Tanner, and A. G. Rinzler, Phys. Rev. B 62, R13 310 (2000).

${ }^{30}$ N. M. Nemes, J. E. Fischer, K. Kamaras, D. B. Tanner, and A. G. Rinzler, in Molecular Nanostructures, edited by H. Kuzmany, J. Fink, M. Mehring, and S. Roth, AIP Conf. Proc. (AIP, New York, 2002), Vol. 633, p. 259.
${ }^{31}$ Kindly provided by T. Pichler.

${ }^{32}$ J. Zhao, J. Han, and J. P. Lu, Phys. Rev. B 65, 193401 (2002).

${ }^{33}$ H. Rauf, T. Pichler, M. Knupfer, J. Fink, and H. Kataura, Phys. Rev. Lett. 93, 096805 (2004).

${ }^{34}$ J. E. Fischer, A. Metrot, P. J. Flanders, W. R. Salaneck, and C. F. Brucker, Phys. Rev. B 23, 5576 (1981).

${ }^{35}$ M. S. Dresselhaus, G. Dresselhaus, and A. Jorio, Annu. Rev. Mater. Res. 34, 247 (2004).

${ }^{36}$ C. Fantini, A. Jorio, M. Souza, L. O. Ladeira, A. G. Souza Filho, R. Saito, G. G. Samsonidze, G. Dresselhaus, M. S. Dresselhaus, and M. A. Pimenta, Phys. Rev. Lett. 93, 087401 (2004).

${ }^{37}$ A. Claye, S. Rahman, J. E. Fischer, A. Sirenko, G. U. Sumanasekera, and P. C. Eklund, Chem. Phys. Lett. 333, 16 (2001).

${ }^{38}$ Z. Chen, X. Du, M. Du, C. D. Rancken, H. Cheng, and A. G. Rinzler, Nano Lett. 3, 1245 (2003). 